\title{
IMPROVING THE USEFULNESS OF OPERATIONAL RADIOSONDE DATA
}

\author{
by Imke Durre, Tony Reale, David Carlson, John Christy, Michael Uddstrom, Melvyn Gelman, \\ Peter Thorne, and CONTRIBUtions from 23 additional Workshop participants
}

A $\mathrm{t}$ the international Workshop to Improve the Usefulness of Operational Radiosonde Data, where delegates represented four continents (for list of participants, see appendix), briefings outlining work related to operational radiosondes highlighted the ever-increasing, and sometimes competing, demands upon and uses of the current radiosonde network. The subsequent discussions led to a broad consensus on findings and recommendations drafted by technical subgroups at the workshop. These findings and recommendations covered: 1) requirements for accuracy, calibration, and corrections; 2) sampling strategies for various applications; and 3) the exchange of and response to information on radiosonde data and metadata. The recommendations both underscore many issues previously

AFFILIATIONS: DURRE-NOAA National Climatic Data Center, Asheville, North Carolina; ReALE-NOAA National Environmental Satellite Data and Information Service, Silver Spring, Maryland; CARLSON-National Center for Atmospheric Research, Boulder, Colorado; CHRISTY-University of Alabama in Huntsville, Huntsville, Alabama; UDDSTROM-New Zealand National Institute for Water and Atmospheric Research, Newmarket, New Zealand; GeLMAN-NOAA Climate Prediction Center, Camp Springs, Maryland; ThorneHadley Centre, Met Office, Berkshire, United Kingdom CORRESPONDING AUTHOR: Imke Durre, National Climatic Data Center, 15I Patton Avenue, Asheville, NC 2880 I

E-mail: imke.durre@noaa.gov

DOI: 10.1 I75/BAMS-86-3-4II

In final form II September 2003

(C2005 American Meteorological Society

\section{WORKSHOP TO IMPROVE THE USEFULNESS OF} OPERATIONAL RADIOSONDE DATA

What: International group of 30 specialists - data users and providers-discuss accuracy requirements, sampling strategies, and other issues in the optimal use of operational soundings

When: II-13 March 2003

Where: Asheville, North Carolina

raised by users and highlight some more recent areas of concern.

\section{ACCURACY, CALIBRATION, AND COR-}

RECTIONS. Background. There is a range of user requirements for radiosonde data in terms of physical measurement accuracy, precision, calibration, and corrections. Reports such as "Guidance regarding how well satellite and in situ sensor capabilities meet user requirements in several application areas" (WMO 2001) list accuracy requirements for different applications. However, no generally agreed upon and documented single set of standards exists that can serve as guidance to users, data providers, and instrument manufacturers. There is a strong need for detailed, justified, and internationally recognized specifications for accuracy, precision, calibration, and corrections for radiosonde measurements. The potential costs involved in meeting new standards and operational methodologies need to be weighed against potential benefits. 
There is also uncertainty in how standards in current WMO documents are applied. For example, the acceptable temperature error is listed as $0.3^{\circ} \mathrm{C}$ for local, synoptic, and climatological use (WMO 2001). However, radiation errors of much greater than $0.3^{\circ} \mathrm{C}$ are common in radiosonde measurements in the upper troposphere and stratosphere. It is common practice for most (but not all) radiosondes to be corrected for those errors. However, it is recognized that these corrections adjust for average conditions, and do not truly correct for the particular conditions for each sounding. Thus, residual temperature errors should be expected to be greater that $0.3^{\circ} \mathrm{C}$. Furthermore, when changes are made at stations in the type of radiosonde that is used, from previously uncorrected to corrected, or between radiosondes with different corrections, spurious temperature shifts of greater than $0.3^{\circ} \mathrm{C}$ are commonly observed.

Accuracy and precision specifications. Revised international accuracy and precision specifications for radiosonde measurements of temperature, humidity, and wind are needed to meet, as effectively as possible, the strict requirements of numerical weather prediction, local weather forecasting, satellite calibration, climate applications, and research.

We recommend that the World Meteorological Organization (WMO) Commission for Instruments and Methods of Observation (CIMO) undertake the task of updating the current requirements for the precision, vertical resolution, and temporal resolution (e.g., number of soundings per day) of measured temperature, humidity, and wind in the CIMO Guide to Meteorological Instruments and Methods of Observation Handbook 8. Representation of all users, manufacturers, and providers is imperative to ensure that the resulting new set of requirements satisfies all communities. Although a single set of accuracy and precision requirements is likely to be necessary as guidance for manufacturers, it may be desirable for the specifications for vertical and temporal resolution to vary among subnetworks of stations, such as the Global Climate Observing System (GCOS) Upper-Air Network (GUAN; WMO 2002) and others mentioned in the "Sampling Strategies" section below.

Upper-tropospheric humidity. Operational measurements of the absolute value and variation of uppertropospheric humidity are presently inadequate for addressing climate variability and change. Some current sensors respond with enough signal in the upper levels to reconstruct a useful profile. Other sensors (e.g., frostpoint mirror) provide exceptionally useful results.
Operational humidity sensors should be developed (required) to respond adequately to variations in the upper troposphere for the purpose of documenting patterns, variations, and long-term changes in humidity, which are critical for understanding climate.

Precipitable water. Current radiosonde precipitable water (PW) measurements contain significant errors. Total column PW can be measured via surface-based remote sensing and provides an overall constraint for PW.

We recommend that there be a move to install global positioning system (GPS) receivers at selected upper-air sites and microwave radiometers on selected ships to provide surface-based constraints on the column PW.

Reference radiosonde. There are candidates for an internationally recognized uniform standard radiosonde (e.g., three-thermistor radiosonde for temperature, chilled mirror for dewpoint) against which operational radiosondes may be compared in order to calculate instrument biases and transfer functions. Such comparison results and transfer functions are useful for the application of bias adjustments and noise reduction.

Systems should be designated by the WMO, so that current and future radiosondes may have their performance characteristics defined by reference to the uniform standards. At least a geographically representative subset of operational radiosondes should have the ability to periodically insert additional sensors, such as the uniform standard sensors, for coincident flight checks.

Data from field research. Field research on radiosonde error characteristics has produced considerable information that could be of significant value to users. Unfortunately, in many cases, this information (e.g., data from multiradiosonde flights) resides with the individual groups who collected it and is not readily available to others.

We recommend that procedures and working groups be established by which important information for determining radiosonde quality characteristics may more easily and effectively be shared and incorporated into operational and research activities through a liaison between the relevant parties. A central international repository (e.g., one of the World Data Centers) for archiving and distributing (via the Internet) all such data, associated documentation, and references should be designated. It is recognized that concerns about confidentiality and commercial sensitivity may require usage restrictions and lead to the un- 
availability of some data. Research utilizing such information should be published in peer-reviewed journals. Radiation error. Variations in solar and infrared radiation impinging on the radiosonde package introduces errors to the temperature measurement. Direct knowledge of these quantities would be useful in reducing such errors (which can easily exceed accuracy and precision specifications). The three-thermistor radiosonde, with its components that each respond differently to the radiation variations, allows correction for radiation variations, although it does not explicitly produce a profile of the radiation quantities.

Temperature sensors should be designed to have high reflectivity and low emissivity to minimize the potential biases. Further research should be conducted into the potential for the routine quality assessment of radiosondes, including a test of radiation response to incoming solar, as well as incoming and outgoing infrared, radiation in a laboratory setting. A sensor measuring solar and infrared radiation should be developed for the purpose of measuring radiation profiles and correcting temperature profiles.

Ultimately, radiation-correction procedures must be developed, which better account for the particular conditions for each sounding, and not the "average" conditions as currently done, and must be accompanied by protocols concerning their application by users to ensure scientific consistency.

On-site calibration. On-site calibration procedures can reduce problems in radiosonde measurements stemming from errors in instrument calibration, but also carry with them additional cost and an increased risk of human error by adding extra complexity to the overall process of launching a radiosonde. Potential calibration procedures include the standard Vaisala ground check employed, for example, by the Australian network and the recording of radiosonde measurements before launch (i.e., the collection of prelaunch data).

Although, in principle, surface calibration could improve accuracy by helping to identify faulty instruments before launch and/or by providing prelaunch data that can be compared to independent surface measurements, the benefits were not judged to be clearly proven for general operational use. It may be most feasible to apply on-site calibration for a subset of stations that are utilized in GUAN or similar networks where long-term precision is vital. We recommend that further research be conducted to assess the benefits of on-site calibration and to determine how they may best be incorporated into operational settings.
SAMPLING STRATEGIES. Background. The network of global radiosonde observations provides an indispensable data resource for an expanding number of critical applications, including

- climate monitoring and climate model validation,

- satellite data analysis (e.g., forward radiative transfer model correction, derivation and validation of products), and

- weather prediction (e.g., specification of initial conditions, local forecaster aids, derivation and validation of products).

All of these present-day applications are, and will continue to be, underpinned by observation programs generating high-quality radiosonde observations of atmospheric parameters. While many of the above applications share common data requirements, conflicting and supplemental needs can also be identified. Of the following findings and recommendations, some relate to more than one application, while others are application specific.

Spatial sampling. Poor spatial sampling over the oceans and many tropical and Southern Hemisphere land areas, as well as the presence of poor-quality data in some of these same regions, act to reduce the utility of the network for almost all actual and potential users.

a) We support the efforts of the CIMO Upper-Air Working Group to assist India in improving its data and encourage the working group to consider expanding its efforts to other countries.

b) We recommend that the Commission for Basic Systems (CBS) consider means for enhancing the atmospheric sounding capabilities of research vessels (and possibly other ships) in order to provide improved data coverage of remote ocean areas.

c) We fully support GCOS efforts to improve coverage and reporting within the Tropics. GCOS should reiterate to providers the importance of continuing soundings in the Tropics, given their importance in the climate change science context.

d) We recommend that interested groups and committees consider the potential benefits and pitfalls of large transnational procurements of radiosondes and ground equipment. Such large procurements could significantly reduce equipment costs.

Consolidation of sampling strategies. Many separate sampling strategies exist both at global and regional levels and for various purposes, that is, the GUAN, sta- 
tions in support of weather prediction, etc. There needs to be a definition, rationalization, and consolidation of these sampling strategies to reduce existing ambiguities. The WMO's Implementation/Coordination Team on Integrated Observing Systems and other relevant committees should consider how to most efficiently rationalize the overall system of sampling strategies.

Selective collocation of radiosonde and satellite observations. Satellite data have become a major source of information driving climate and weather applications. A continuous, robust and traceable sample of global, radiosonde observations that are coincident with satellite overpass times is required for the validation of the measurements, processing algorithms, and derived products from operational and research polarorbiting satellites.

We recommend that relevant working groups [e.g., the CBS Expert Team on Observational Data Requirements and Redesign of the Global Observing System (GOS)] consider establishing a sampling strategy and requirements for a geographically diverse subset of sites where well-characterized radiosondes will be launched at satellite overpass times. This subnetwork should include observations from small islands and ships, selected Atmospheric Radiation Measurement (ARM) sites, all primary Network for the Detection of Stratospheric Change (NDSC) sites, operational observing stations, and a selection of sites where additional surface-based observing systems (e.g., ground-based GPS precipitable water sounders, ship-based microwave radiometers, ozonesondes, and rocketsondes) are deployed. Site selection must also consider existing climate and numerical weather prediction (NWP) strategies.

Climate monitoring needs. It is important from a climate monitoring perspective to maintain as many existing long-term records as possible, while minimizing changes in instrumentation and practices. The precision of a bias calculation for a specific radiosonde due, for example, to a change in instrumentation, depends on the length of the individual homogeneous segment of measurements. The presence of adequate observations from dual-radiosonde flights may reduce any minimum requirement for the length of a homogeneous segment.

We recommend that radiosonde climate applications groups, including GCOS, decide upon a list of those stations currently reporting with sufficiently long records to be considered useful in a climate context. It may be advantageous to divide this list into two or three sub-lists, for example, tier 1, consisting of those that are already GUAN and Lanzante-KleinSeidel (2003) stations; tier 2, consisting of an additional subset of well-spaced good stations (that could also replace GUAN stations if needed); and tier 3, the remaining long-term stations (and candidate for selective launch coincident with satellite overpass). The resulting lists should be channeled to providers through GCOS, who should consider giving explicit guidance regarding the minimization of changes at these sites while recognizing that some change is both inevitable and desirable. The specifics of such guidance may differ for different tiers, reflecting their relative importance. GCOS is further requested to consider the adequacy of dual-radiosonde flights and formulate specific recommendations considering, in particular, the length of time and degree of spatial sampling required for dual-launch systems to be adequate for climate study purposes.

Targeted launches. The ability to undertake weathersensitive targeted launches (globally) at short notice from current observing sites would greatly enhance the usefulness of radiosondes to NWP. Such targeted launches are the subject of ongoing work in the Observing System Research and Predictability Experiment (THORPEX).

We support the efforts of THORPEX to implement the concept of targeting, whereby "observations are utilized and/or supplemented where and when their impact on the quality of the analyses and forecasts is largest" (THORPEX, 2003).

Multisensor platforms. Multisensor platforms over both land and ocean could yield significant synergies.

We recommend that the proper CBS working groups consider the experimental installation of other surface-based sounding equipment coincident with a selection of land- and ship-based radiosonde sounding sites, taking into account the potential for synergy not just with radiosondes, but also with satellite observations. For example, high-quality ground-based GPS receivers at selected upper-air sites as well as shipbased microwave radiometers would provide surfacebased constraints on the column precipitable water.

Sampling of the stratosphere. There is a growing requirement for a better sampling of the middle to upper stratosphere for both climate and satellite operations.

Appropriate sampling strategies concerning the vertical resolution and extent of radiosonde observations in the middle to upper stratosphere need to be defined by consultation between relevant user groups and committees. It is recommended that the sampling 
strategies include a specification of the minimum percentage of flights that will be required to reach a specified minimum height (e.g., $5 \mathrm{hPa}$ ).

0000// 200 UTC observations. Both weather prediction and climate monitoring require 0000 and 1200 UTC soundings to (begin to) sample the diurnal cycle and to maintain continuity with the historical record.

We recommend that, as certain sites are selected for radiosonde launches coincident with satellite overpass times, a 0000/1200 UTC sampling approach be maintained wherever possible. This is most important for GUAN stations and other stations with long records used for climate monitoring.

EXCHANGE OF AND RESPONSE TO INFORMATION: DATA INTEGRITY, METADATA, REAL-TIME MONITORING, AND FEEDBACK. Background. All users of radiosonde data rely to some extent upon accurate information on observational practices. Metadata that are currently available are insufficient for many users, particularly those involved in climate research where knowledge of changes in instrumentation/observational practice is imperative in constructing long-term homogeneous series. The associated issues of traceability and reversibility, that is, the ability to both trace and reverse the processing steps applied to the original data, are also important to most users.

Data integrity is important in all radiosonde applications, but can easily be compromised during collection, transmission, processing, and storage. It is generally recognized that efforts should be made to continue to actively address and minimize this longstanding challenge A wealth of information that is relevant to the real-time monitoring of data quality and metadata is collated both by analysis centers and the WMO. However, there was general agreement among workshop participants that there are current inefficiencies in the utilization of this information by providers, equipment manufacturers, and radiosonde data end users.

Metadata. Station history information is contained in databases residing at various data centers as well as in WMO-maintained lists and catalogues, such as Publication 9, Volume A. There exists a need to fill in and frequently update records of upper-air station history information as well as to reconcile the metadata records maintained by various centers. Efforts are currently underway to obtain up-to-date station history information through a Web-based survey of WMO member countries. a) Designated centers [e.g., National Climatic Data Center (NCDC) and the Hadley Centre] should undertake a concerted, cooperative program for resolving problems with historical metadata, particularly focusing on pinning down the timing of changes. They should also provide high-resolution metadata in the future using data held in coded radiosonde messages to complement research-quality radiosonde datasets. The resulting historical and real-time metadata should be made widely available to users in a number of easily accessible forms.

b) We recommend that station lists such as WMO Publication 9, Volume A, be updated consistently for all parts of the world and made available on the Internet in a timely manner to accurately reflect recent changes in station location, instrumentation, corrections applied operationally, and other metadata.

Content of radiosonde messages. The current coded message includes space for the actual launch time, radiation bias code, wind-finding equipment used, and other metadata, but this information is not provided by all stations. Once messages are transmitted in binary universal form for representing meteorological data (BUFR), the possibility also exists for including additional information that cannot be transmitted in the current message format.

The proper WMO working group should provide guidance to operators that encourages them to include as much information as possible in the message to make the data more useful to a large range of applications. The WMO should consider that the following are deemed to be highly desirable for inclusion in future BUFR-formatted messages:

- station name;

- latitude, longitude, and time at every level;

- height of the surface level;

- relative humidity at all levels;

- prelaunch data;

- high-resolution data rather than solely mandatory and significant levels;

- elapsed time.

Reversibility and traceability. There is a need for reversibility and traceability. Experiences of some users have pointed towards the presence of errors in, for example, operationally applied radiation correction factors, which can, in theory, easily be corrected. Increased traceability and reversibility would simplify the implementation of such back corrections. 
Data processing centers should be encouraged to keep data that are not quality controlled as well as, if possible, undecoded radiosonde messages. The proper WMO working group should consider whether it is feasible for sites to transmit or otherwise retain both uncorrected and radiation-corrected data.

Quality control. The potential exists for increased communication among relevant groups and individuals regarding decoding and quality-control $(\mathrm{QC})$ issues.

A process should be established for exchanging decoding and QC methods and tools. That process should consist of

- a common Web site, including test datasets, sharable code, and plots of soundings in which data values were rejected that could serve as feedback to the site operators;

- a workshop to exchange methods;

- a formal intercomparison of QC systems similar to existing model intercomparison projects.

This exchange could lead to the development of automated QC analysis indicators that could provide further feedback between monitoring centers and sites and assist in removing discrepancies between the blacklists maintained by the various centers.

Information exchange. There are some weaknesses and inefficiencies in the current system management of information exchanges among users, monitoring centers, and operational sites.

It follows that:

- There should be a larger number of regional radiosonde workshops that provide training to the operators and managers of radiosonde sites. Attempts should be made to raise awareness of the importance of radiosonde data and metadata. Operators should discuss options for incentives to encourage high performance of all individual radiosonde sites, learning from the experiences of the National Weather Service (NWS). The designation of a station for specific network processes (e.g., GUAN) may also act as an incentive to station operators.

- Expert WMO groups should improve the process of resolving deficiencies in the global radiosonde network and provide a clearer mechanism for input to those processes from customers and the scientific community.

- The Commission for Basic Systems should consider establishing an expert team focused on radiosondes.
- WMO and analysis centers should consider, through consultation with the user community, how to make the crucial information more accessible.

CONCLUSIONS. We recognize that implementation of the above recommendations hinges upon actions by numerous groups. We urge CBS and CIMO to make a review of accuracy requirements and sampling strategies a priority. We further encourage interested scientists to pursue the research questions posed by several of the recommendations and funding agencies to provide the resources for such research. To initiate the implementation process, this report is being submitted to specific contacts in various organizations [e.g., WMO, National Oceanic and Atmospheric Administration (NOAA)]. Workshop participants will monitor and/or play an active part in the progress of the recommendations and will decide at a later date whether a follow-up meeting or further action is warranted.

ACKNOWLEDGMENTS. This workshop was funded by the Climate Data and Detection element of the NOAA Climate and Global Change Program and hosted by the NOAA National Climatic Data Center. John Christy was supported by NOAA Office of Global Programs Grant NA96GP0396. Peter Thorne was supported by the United Kingdom's Department of the Environment, Food, and Rural Affairs under contract PECN7/12/37, and through their contribution, this report is Crown Copyright.

\section{APPENDIX: PARTICIPANTS}

Charles Anderson

Canadian Meteorological Centre

charles.anderson@ec.gc.ca

Bradley Ballish

NOAA Environmental Modelling Center

bradley.ballish@noaa.gov

Kautsubha Bhattacharya

NCMRWF

Department of Science and Technology

Government of India

batta@ncmrwf.gov.in

Michael Berechree

Australian Bureau of Meteorology

m.berechree@bom.gov.au

Carl A. Bower Jr.

Observing Systems Division

NOAA National Weather Service

carl.bower@noaa.gov 
David Carlson (working group chair)

Atmospheric Technology Division

National Center for Atmospheric Research

dcarlson@atd.ucar.edu

John Christy (working group chair)

Earth System Science Center

University of Alabama

christy@nsstc.uah.edu

Imke Durre (workshop co-chair)

NOAA National Climatic Data Center

imke.durre@noaa.gov

Joseph Facundo (session chair)

Observing Systems Division

NOAA National Weather Service

joseph.facundo@noaa.gov

Antonio Garcia-Mendez

European Centre for Medium-Range Weather

Forecasts

a.garcia@ecmwf.inta.garcia@ecmwf.int

Melvyn Gelman (session chair)

NOAA Climate Prediction Center

melvyn.gelman@noaa.gov

Larry J. Griffin

NOAA National Climatic Data Center

Larry.J.Griffin@noaa.gov

Brian D. Jamison

NOAA Forecast Systems Laboratory

brian.d.jamison@fsl.noaa.gov

Magnus Lindskog

Swedish Meteorological and Hydrological Institute

magnus.lindskog@smhi.se

Larry McMillin

NOAA National Environmental Satellite Data and

Information Service

larry.mcmillin@noaa.gov

John Nash (WMO representative, session chair)

Met Office

john.nash@metoffice.com

Thomas C. Peterson

NOAA National Climatic Data Center

thomas.c.peterson@noaa.gov
Tony Reale (workshop co-chair)

Satellite Applications and Research

NOAA National Environmental Satellite Data and

Information Service

tony.reale@noaa.gov

Christopher Redder

Data Assimilation Office

NASA Goddard Space Flight Center

credder@dao.gsfc.nasa.gov

Hank Revercomb

Space Science and Engineering Center

University of Wisconsin

hankr@ssec.wisc.edu

Francis J. Schmidlin

Wallops Flight Facility

NASA Goddard Space Flight Center

fjs@osb1.wff.nasa.gov

Russell Schwanz

Fleet Numerical Meteorology and Oceanography

Center

U.S. Navy

russell.schwanz@fnmoc.navy.mil

Peter Thorne (rapporteur)

Hadley Centre

Met. Office

peter.thorne@metoffice.com

Dave Tobin

Space Science Engineering Center

University of Wisconsin

dave.tobin@ssec.wisc.edu

Michael J. Uddstrom (working group chair)

New Zealand National Institute of Water and

Atmospheric Research

m.uddstrom@niwa.cri.nz

Junhong Wang

Atmospheric Technology Division

National Center for Atmospheric Research

junhong@ucar.edu

Werner Wergen (session chair)

Deutscher Wetterdienst

werner.wergen@dwd.de 
Ed R. Westwater

CIRES

University of Colorado

ed.r.westwater@noaa.gov

John White

U.S. Air Force Combat Climatology Center john.white@afccc.af.mil

\section{REFERENCES}

Lanzante, J. R., S. A. Klein, and D. J. Seidel, 2003: Temporal homogenization of monthly radiosonde temperature data. Part 1: Methodology. J. Climate, 16, 224-240.
Shapiro, M. A., and Thorpe, A. J., 2002: The observingsystem research and predictability experiment THORpex. Program overview, 16 pp. [Available online at www.mmm.ucar.edu/uswrp/programs/ thorpex.html.]

WMO, 2001: Statement of guidance regarding how well satellite and in situ sensor capabilities meet WMO user requirements in several application areas. WMO/TD 1052, Sat-26, WMO, Geneva, Switzerland, 48 pp. [Available online at www.wmo.int/hinsman/ SAT-26\%20SOG\%202001and\%20Annex\%20A.doc.] , 2002: Guide to the GCOS surface and upper-air networks: GSN and GUAN. WMO/TD 1106, GCOS73, WMO, Geneva, Switzerland, 37 pp. [Available online at www.wmo.ch/web/gcos/Publications/gcos73.pdf.] 